\title{
Tuberculosis activa en una cohorte de reclusos infectados por VIH en una cárcel de la Ciudad de México: características clínicas y epidemiológicas
}

\author{
Christian Hernández-León, MC,(I) Florentino Badial-Hernández, MC, M en C, M en FS, ${ }^{(3)}$ Alfredo Ponce-de-León, MC,(I) \\ Juan G Sierra-Madero, MC,(I) Areli Martínez-Gamboa, QFB, Dr en C, (I) Brenda Crabtree-Ramírez, MC,(I) \\ Sergio Bautista-Arredondo, M en C, ${ }^{(2)}$ Adrián González-Aguirre, MC, (I) María de Lourdes Guerrero-Almeida, MC, M en C,(1) \\ J Miriam Bobadilla del Valle, QFB, Dr en C,(1) Andrea González-Rodríguez, MC, ${ }^{(3)}$ José Sifuentes-Osornio, MC. ${ }^{(1)}$
}

\begin{abstract}
Hernández-León C, Badial-Hernández F, Ponce-de-León A, Sierra-Madero JG, Martínez-Gamboa A, Crabtree-Ramírez B, Bautista-Arredondo S, González-Aguirre A, Guerrero-Almeida ML, Bobadilla del Valle JM, González-Rodríguez A, Sifuentes-Osornio J. Tuberculosis activa en una cohorte de reclusos infectados por VIH en una cárcel de la Ciudad de México: características clínicas y epidemiológicas. Salud Publica Mex 20 12;54:57 I-578.
\end{abstract}

\section{Resumen}

Objetivo. Determinar las características clínicas y epidemiológicas de los casos con tuberculosis (TB) activa en población de cárceles con VIH, que se ve especialmente afectada por ambas epidemias. La infección por VIH incrementa significativamente la probabilidad del desarrollo de TB. Material y métodos. Se realizó un estudio de cohorte en sujetos infectados por $\mathrm{VIH}$ e internados en un reclusorio de la Ciudad de México. Resultados. Se encontraron 172 pacientes con $\mathrm{VIH}, 28$ con TB activa (I6.3\%) - 2I (I2.2\%) con afección pulmonar - con una tasa de incidencia de 7.7 por 100 sujetos/año para TB activa y de 4.7 por 100 sujetos/ año para TB pulmonar. No se encontró drogorresistencia. Dieciocho aislados fueron tipificados por RFLP, con una tasa de transmisión calculada de II\%. Conclusión. Se encontró una prevalencia de TB en esta población mil veces superior a la observada en la población general y datos sugerentes de transmisión al interior de la cárcel.

Palabras clave: tuberculosis; prisiones; VIH; epidemiología; México

\author{
Hernández-León C, Badial-Hernández F, Ponce-de-León A, \\ Sierra-Madero JG, Martínez-Gamboa A, Crabtree-Ramírez B, \\ Bautista-Arredondo S, González-Aguirre A, \\ Guerrero-Almeida ML, Bobadilla del Valle JM, \\ González-Rodríguez A, Sifuentes-Osornio J. \\ Active tuberculosis in a cohort of HIV-infected inmates \\ in a prison in Mexico City: clinical and \\ epidemiological characteristics. \\ Salud Publica Mex 20 12;54:57 I-578.
}

\begin{abstract}
Objective. To determine the clinical and epidemiological characteristics of prison inmates with active tuberculosis in HIV-positive prison populations. Materials and methods. We conducted a cohort study in HIV-infected subjects in a prison in Mexico City, with the aim of determining clinical and epidemiological characteristics of cases with active TB. Results. We detected 172 HIV infected inmates and TB in 28 of them (16.3\%) - 2I (12.2) with pulmonary TB - with an incidence rate of $7.7 / 100$ persons/year for active TB and 4.7/100 persons/year for pulmonary TB. No drug resistance was found. Two clusters ( 4 and 2 subjects) were observed after RFLP-typing of 18 isolates, with a transmission rate of II\% by molecular and clinical analysis. Conclusions. The prevalence of active TB was found to be a thousand times greater than in the general population. Evidence of transmission inside the prison was also found.
\end{abstract}

Key words: tuberculosis; prisons; HIV; epidemiology; Mexico

(I) Instituto Nacional de Ciencias Médicas y Nutrición Salvador Zubirán. México.

(2) Instituto Nacional de Salud Pública. México.

(3) Clínica Especializada Condesa. México.

Fecha de recibido: 29 de noviembre de $201 \mathrm{I}$ • Fecha de aceptado: 28 de agosto de 2012

Autor de correspondencia: Dr. José Sifuentes Osornio. Laboratorio de Microbiología Clínica, Instituto Nacional de Ciencias Médicas y Nutrición Salvador Zubirán. Vasco de Quiroga I5, colonia Sección XVI. I 4000 Delegación Tlalpan, México, DF, México.

Correo electrónico: sifuentesosornio@gmail.com 
$\mathrm{H}_{\mathrm{t}}$ asta un tercio de la población mundial está infectada por Mycobacterium tuberculosis ${ }^{1}$ y $90 \%$ de ella tiene la infección en su forma latente (TBIL). ${ }^{2} \mathrm{La}$ probabilidad de que la TBIL se active incrementa significativamente cuando el individuo está coinfectado por el virus de la inmunodeficiencia humana (VIH), con una tasa anual de $10 \%$. $^{3}$ Se ha observado que la tuberculosis (TB) es un problema de salud en el ámbito carcelario, ,,5 con rangos de incidencia y prevalencia en prisiones que exceden de manera significativa a los observados en la población general. ${ }^{6,7}$ Del mismo modo, los rangos de coinfección TB-VIH se han encontrado más elevados en comparación con la población general. ${ }^{8,9}$ La prevalencia de TB activa en la población mexicana es de 16.3 por cada 100000 habitantes. ${ }^{10}$ Sin embargo, la información acerca de esta infección en prisiones de México es escasa.

En febrero de 2009, la Clínica Especializada Condesa (CEC), el Instituto Nacional de Ciencias Médicas y Nutrición Salvador Zubirán (INNSZ) y el Instituto Nacional de Salud Pública (INSP) iniciaron un programa de atención para internos VIH+ del Sistema Penitenciario de la Ciudad de México (SPCM) en el Centro de Readaptación Social Masculino de Santa Martha Acatitla (CRSM-SMA). En este centro se han concentrado los internos infectados por $\mathrm{VIH}(\mathrm{VIH}+)$ que requieren tratamiento. Entre febrero de 2009 y diciembre de 2010 el programa atendió a 172 internos (figura 1). Además, 105 internos VIH+ detectados por un protocolo del INSP que aún se encuentra en curso continuaban en sus centros por diferentes razones.

La población del CRSM-SMA fue de 2553 internos, 108 de ellos (4.23\%) VIH+ en diciembre de 2010. Todo esto indica un riesgo elevado para la transmisión y desarrollo de TB activa en este centro. Por ello, se llevó a cabo este estudio cuyo objetivo fue determinar las características clínicas y epidemiológicas de los casos de TB en internos VIH+ del CRSM-SMA.

\section{Material y métodos}

Diseño. Estudio de cohorte retrospectivo realizado en sujetos VIH+ concentrados en el CRSM-SMA y anidado en el proyecto "Intervención sanitaria en Centros Penitenciarios/Detección, atención y prevención de enfermedades en Centros Penitenciarios de la Ciudad de México (CM)", iniciativa hecha por INSP, INNSZ y el gobierno de la CM. Las principales variables de salida fueron TB activa y la sospecha de transmisión reciente dentro de la cárcel por datos epidemiológicos clínicos y moleculares. El estudio fue evaluado y aprobado por los comités de investigación y de ética de las instituciones participantes. Se obtuvo el consentimiento informado de todos pacientes en prisión entre noviembre de 2010

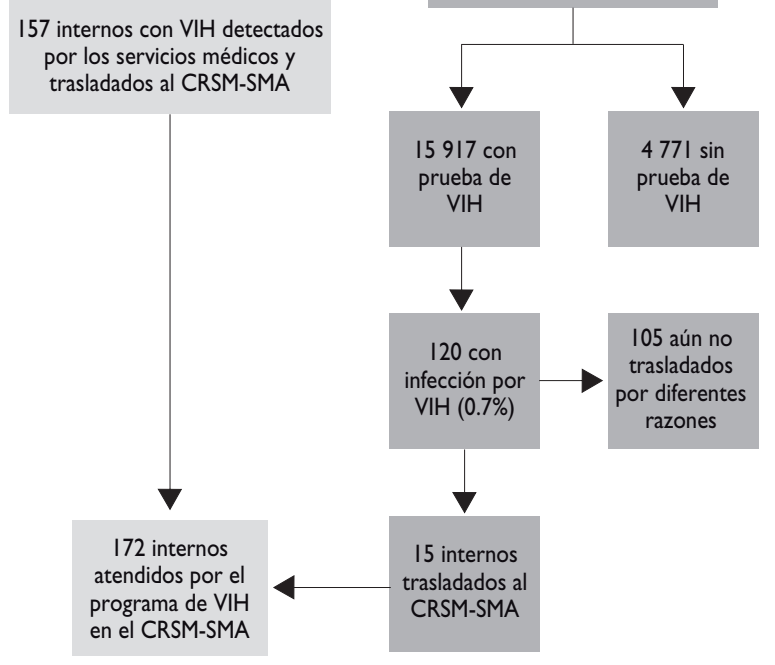

Población del género masculino del sistema penitenciario de la Ciudad de México (diciembre, 2010). En diciembre de 2010 se encontraba en marcha un protocolo de prevalencia de VIH en el Sistema Penitenciario de la Ciudad de México que, hasta ese momento, había incluido a tres centros. De los internos detectados por ese protocolo, 15 habían sido trasladados a la Penitenciaría de Santa Martha Acatitla (CRSM-SMA) para su atención por el Programa de VIH. Los otros 157 fueron detectados por los Servicios Médicos y por la Clínica Condesa y trasladados al CRSM-SMA

Figura I. Población del género masculino del sistema penitenciario del Distrito Federal. Diciembre, 2010

y febrero de 2011. Con la aprobación pertinente se incluyeron los casos de los pacientes previamente liberados o muertos.

Población y sitio de estudio. Todos los internos VIH+ del CRSM-SMA fueron atendidos por el programa y, a su ingreso, fueron sometidos al protocolo de detección de TB (historia clínica y exploración física, radiografía de tórax y baciloscopía en tres muestras de expectoración). Posteriormente, en los internos con datos compatibles con TB pulmonar -tos con expectoración durante más de dos semanas, radiografía de tórax sugerente- se tomaron cultivos de expectoración, de lavado bonquioalveolar o de aspirado endotraqueal. En aquellos pacientes con datos sugerentes de TB extrapulmonar en quienes no se hubiera demostrado previamente TB pulmonar se solicitó cultivo y estudio histopatológico de las muestras clínicas pertinentes. Todos los pacientes incluidos en la cohorte fueron citados a consultas de seguimiento para el control de la infección por VIH 
-cada 3 a 6 meses como mínimo, dependiendo de cada paciente- o cuando el paciente solicitaba la consulta; si éste presentaba algún síntoma o signo sugerente de $\mathrm{TB}$, se repetía el protocolo de detección. Se siguieron las recomendaciones de la NOM-006-SSA2-1993 ${ }^{11}$ para las definiciones de TB pulmonar y extrapulmonar, así como en las variables relacionadas con el tratamiento (curación, abandono, recaída y traslado). Durante su estancia en prisión el tratamiento fue administrado bajo el esquema TAES.

Todos los internos $\mathrm{VIH}+$ habitan en grupos de cuatro o cinco en estancias diseñadas para dos o tres personas. Tanto los dormitorios como los pasillos de acceso carecen de ventanas o puertas -fuera de la puerta principal del pasillo- para iluminación natural o ventilación.

Procedimientos. Los datos clínicos se obtuvieron por revisión del expediente clínico $(n=10)$ o por entrevista directa $(n=18)$. Se realizó revisión del expediente radiográfico por un médico radiólogo del INNSZ.

Microbiología. El procesamiento de las muestras se llevó a cabo mediante técnicas estandarizadas (tinción de Ziehl-Neelsen, cultivo en medios de LowensteinJensen y MGIT), ${ }^{12}$ y la identificación y las pruebas de susceptibilidad antimicrobiana con métodos aceptados internacionalmente. ${ }^{13}$ Los aislados de $M$. tuberculosis fueron genotipificados mediante la técnica de análisis del polimorfismo en la longitud de los fragmentos de restricción (RFLP) utilizando como sonda la secuencia de inserción 6110 (IS6110) ${ }^{14}$ y por espoligotipificación si el aislado mostraba un patrón de RFLP con menos de seis bandas. ${ }^{15}$ Se consideraron como aislados pertenecientes a un conglomerado de transmisión reciente cuando compartían todas y cada una de las copias de IS6110, cuya posición fuera idéntica. Los aislados con patrón de RFLP diferente se consideraron como únicos.

Análisis estadístico. Se utilizaron los programas Excel para Windows 2007 para la captura de los datos y STATA 8.0 para su análisis. Se calcularon las prevalencias y las tasas de incidencia. Para el análisis de los datos se utilizaron medidas de tendencia central (media y mediana) y de dispersión (desviación estándar, rangos) para las variables de tipo cuantitativo, en tanto que para las variables de tipo cualitativo se utilizaron medidas de frecuencia y proporciones.

\section{Resultados}

Durante el periodo de estudio, se identificaron 172 internos VIH+ con un seguimiento en conjunto de 2137 meses-paciente (175.7 años-paciente o 64124 días-paciente, mediana 292.5 días y rango intercuartilar 128.0 a 292.5) (cuadro I). Se diagnosticaron 28 casos de TB activa (atendidos por el programa de VIH en las cárceles del DF); 21 fueron TB pulmonar, ocho de ellos con evidencia de infección diseminada, y siete más TB extrapulmonar sin evidencia de compromiso pulmonar (figura 2). Así, la prevalencia de TB activa en cualquiera de sus presentaciones entre la población $\mathrm{VIH}+$ del SPCM fue de 28/277, es decir, 10.11 por cada 100 personas, mientras que la prevalencia de TB pulmonar -con o sin evidencia de infección diseminada- en la misma población fue de $21 / 277$, es decir, 7.58 por cada 100 personas. Al considerar únicamente a los internos VIH+ del CRSM-SMA -sometidos al protocolo de detección de TB-las prevalencias fueron de $28 / 172$, es decir, 16.28 por cada 100 pacientes y 21/172 ó 12.21 por cada 100 personas, respectivamente.

Las tasas de incidencia fueron calculadas con base en la cohorte de pacientes VIH+ asintomáticos al momento de la inclusión y seguidos. Para este análisis se sustrajo el tiempo de seguimiento de los 15 pacientes que ingresaron a la cohorte con TB activa. La incidencia de TB activa fue de 13 casos en 168.7 años/persona (13/2053.1 meses/persona), es decir, una tasa de incidencia de 7.7 casos/ 100 sujetos/año o 6.3 casos/1000 sujetos / mes. En el caso de TB pulmonar fueron de ocho casos en 168.7 años / persona (8/2053.1 meses / persona) y de 4.7 casos / 100 sujetos / año o 3.9 casos / 1000 sujetos / mes, respectivamente.

Fue un grupo joven con una mediana de edad de 33.5 años; 27 pacientes $(96.5 \%)$ con estancia previa en reclusorios. Los hallazgos clínicos más frecuentes fueron: pérdida de peso $(92 \%)$, tos $(88 \%)$, diaforesis $(84 \%)$, fiebre $(78.6 \%)$, disnea $(56.5 \%)$ y dolor torácico (36.4\%); otros hallazgos fueron linfadenopatía (81.5\%), signos pulmonares $(52.2 \%)$ y hepato o esplenomegalia (29.2\%). En 15/28 (53.5\%) casos se hizo el diagnóstico de infección por VIH y de TB de manera simultánea. Los pacientes presentaron síntomas sugestivos por tiempo variable previo al diagnóstico con una mediana de 42 días (rango 10-96). Los otros 13 casos (46.5\%) fueron diagnosticados con infección por VIH 41 meses (mediana) antes del inicio de los síntomas de TB (rango 14-188 meses); tres de éstos recibían tratamiento antirretroviral (ARV) al momento del diagnóstico de TB, todos en falla virológica por mal apego terapéutico; cinco de los 10 restantes eran vírgenes a ARV.

La localización más frecuente de la TB fue pulmonar, con afección pulmonar exclusiva en 13 casos $(46.4 \%)$ y formas diseminadas con afección pulmonar en otros ocho $(28.6 \%)$, y los siete restantes $(25 \%)$ fueron extrapulmonares (figura 2). Se revisó la radiografía de tórax de 23 pacientes ( $20 / 21$ con afección pulmonar $\mathrm{y}$ $3 / 7$ con TB extrapulmonar); los infiltrados alveolares fueron los cambios más frecuentes $(30.4 \%)$, seguido de patrón miliar (13\%) e intersticial (4.3\%); también se 
Cuadro I

Características sociodemográficas y clínicas de los internos con tuberculosis activa en UNA COHORTE de reclusos infectados por VIH en una cárCel de la Ciudad de MéXico. Noviembre de 2010-febrero 201 I

Características

Pacientes con tuberculosis activa, $n=28$ (\%)

Edad, años [mediana (rango)]

33.5 años $(20$ a 68$)$

Escolaridad

Primaria o menor

$9 / 24(37.5 \%)$

\begin{tabular}{lc}
\hline Secundaria & $9 / 24(37.5 \%)$ \\
\hline Preparatoria & $1 \mathrm{I} / 24(45.8 \%)$ \\
& $4 / 24(16.7 \%)$ \\
Consumo de tabaco, previo o actual & $23 / 27(85.2 \%)$ \\
\hline IT [media (DE)] & $4.9(6.1)$ \\
& $26 / 27(96.3 \%)$ \\
\hline Consumo de alcohol, previo o actual & $12(46.1 \%)$ \\
\hline Mayor o igual a 40 g/día & $9(34.6 \%)$ \\
\hline Menor de 40 g/día & $5(19.2 \%)$
\end{tabular}

Consumo de drogas, previo o actual

\begin{tabular}{lc} 
Negado & $4 / 26(15.4 \%)$ \\
\hline Endovenosas & $6 / 26(23.1 \%)$ \\
\hline Inhaladas & $22 / 26(84.6 \%)$
\end{tabular}

Cicatriz de BCG

$17 / 2 \mid(80.9 \%)$

$\begin{array}{ll}\text { Tratamiento antituberculosis previo } & 3 / 28(10.7 \%)\end{array}$

Tiempo de estancia en prisión previo al inicio de síntomas, meses [mediana (rango)]

Intervalo entre inicio de síntomas y tratamiento, meses [mediana (rango)]

$43.5(2-118)$

CD4 al diagnóstico [mediana (rango)]*

$3(1-18)$

$\mathrm{CV}$ al diagnóstico, log [mediana (rango)]+

$113(12$ a 506$)$

Tratamiento antirretroviral al diagnóstico

$5.36(2.86-6.87)$

$3 / 28(10.7 \%)$

* CD4, linfocitos CD4 en sangre periférica; + CV, Carga de VIH en sangre periférica

observaron cavernas (13\%), nódulos múltiples $(4.3 \%)$, nódulo pulmonar solitario (13\%), derrame pleural $(13 \%)$, cambios cicatriciales $(8.7 \%)$. En $5 / 23$ pacientes la radiografía fue normal $(21.7 \%)$, dos de ellos con cultivo positivo en expectoración y los otros tres con afección puramente ganglionar.

En los 28 pacientes se inició tratamiento antiTB; 26 recibieron tratamiento primario $(92.9 \%)$ y dos retratamiento primario $(7.1 \%)$, uno por abandono y el otro por fracaso. Trece se encontraban con tratamiento al término del estudio (46.4\%); otros siete lo completaron ( $25 \%)$, todos con curación; ocho no lo completaron (28.6\%), cinco por abandono, ya que fueron liberados y no acudieron a la clínica de referencia, y otros tres fallecieron, dos por TB y otro por probable toxoplasmosis cerebral.
Veintitrés pacientes $(82.1 \%)$ recibieron tratamiento ARV concomitante con el tratamiento antiTB. De los cinco que no lo recibieron, tres rehusaron tomarlo de manera simultánea y lo iniciaron al término del tratamiento antiTB; uno más falleció a los cuatro días del inicio de tratamiento y el otro fue liberado y perdido para seguimiento. Los esquemas utilizados fueron tenofovir + emtricitabina + efavirenz en 16 pacientes y tenofovir + emtricitabina + zidovudina + abacavir en siete, con dos fallas virológicas documentadas, una en cada grupo; 9/23 pacientes con ARV desarrollaron síndrome inflamatorio de reconstitución inmune (SIRI) asociado con $\mathrm{TB}$, cuatro desenmascarante y cinco paradójico.

En seis pacientes no fue posible procesar muestra para cultivo por cuestiones logísticas. Se obtuvieron 


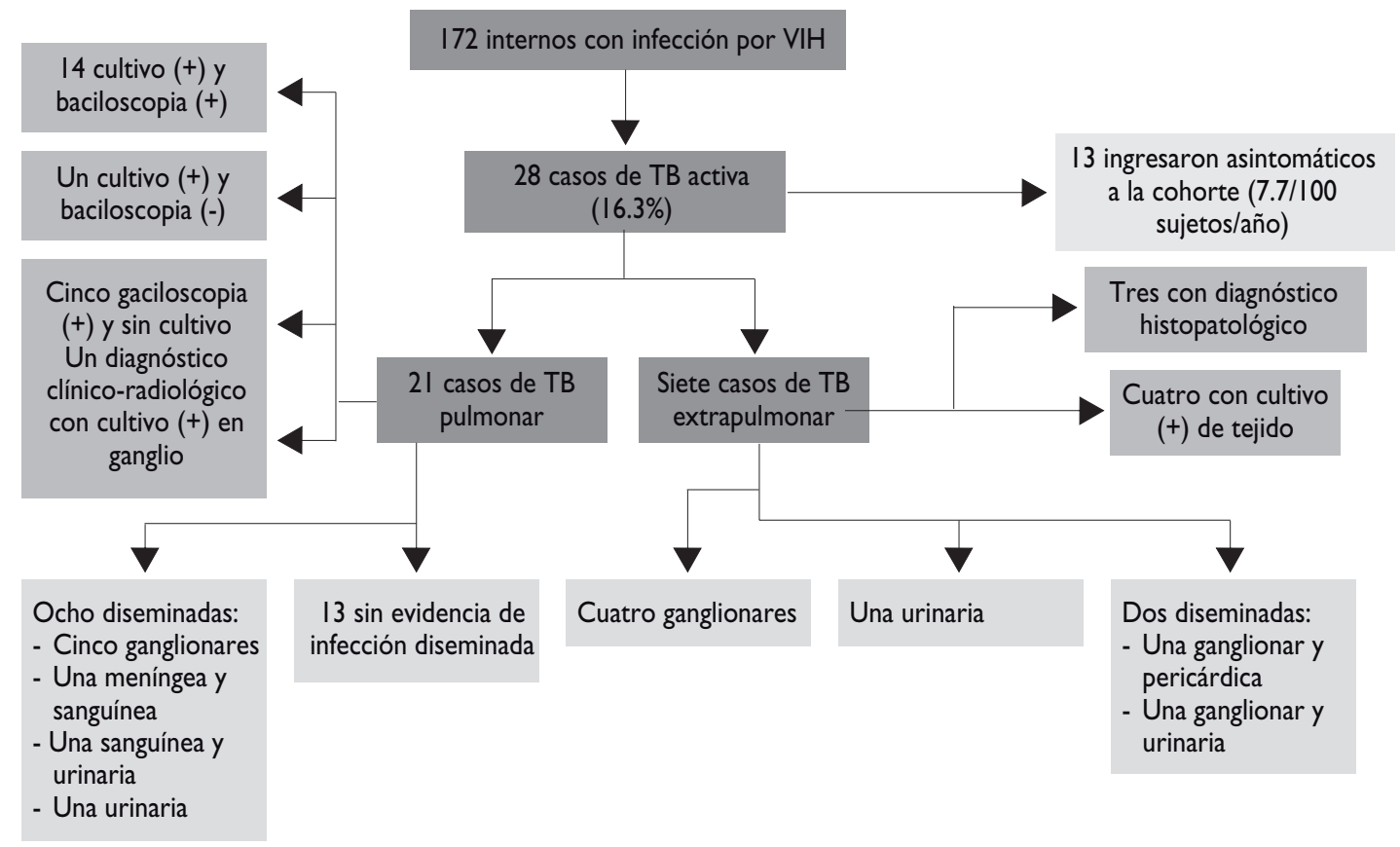

Figura 2. Internos con infección por VIH del sistema penitenciario del Distrito Federal atendidos entre febrero 2009 Y DICIEMBRE 2010

20 cultivos positivos (figura 2), 15 de expectoración y cinco de tejido o de otras muestras, y dos cultivos negativos; 18 aislados fueron genotipificados, $12(66 \%)$ con un patrón único de IS6110 y seis (33\%) formaron dos conglomerados, uno con cuatro aislados (8 copias de IS6110) -que se confirmó por espoligotipificacióny el otro con dos aislados (10 copias de IS6110) (figura 3). De los dos aislados no tipificados, uno provenía de un cultivo mixto con M. tuberculosis y M. kansasii y el otro fue M. bovis. Por otro lado, se realizó segunda tipificación por espoligotipificación en el aislado 19 por mostrar menos de seis copias de IS6110 y en los aislados 13 y 14 por presentar más de 15 copias. El aislado 19 fue tipificado como variante T1, y los aislados 13 y 14 como pertenecientes a las familias Manu y LAM9, respectivamente.

En el primer conglomerado, el primer caso inició síntomas dentro del CRSM-SMA en septiembre de 2009 e inició tratamiento al mes siguiente (figura 4). Los casos 2 y 3 ingresaron al CRSM-SMA en abril y mayo de 2010, respectivamente; desarrollaron síntomas en junio. En cambio, el caso 4 inició síntomas en otro reclusorio (donde ninguno de los otros tres sujetos había estado) y es trasladado al CRSM-SMA en julio de 2010. La única relación epidemiológica entre los cuatro individuos fue que los casos 1 y 3 trabajaban en el servicio sexual antes de ingresar a prisión en la Ciudad de México y el caso 4 reportó ser usuario del servicio sexual en la misma delegación. En el conglomerado 2, el caso 1 inició síntomas cuando convivía en el dormitorio con el caso 2, quien desarrolló síntomas ocho meses después de la muerte del primero. Así, por la presencia de dos genotipos idénticos que incluyeron a seis de los 18 aislados tipificados, es decir, un caso índice para cada conglomerado y cuatro casos secundarios, se puede decir que hubo transmisión reciente y progresión rápida en los cuatro casos secundarios $(22.2 \%)$.

\section{Discusión}

En este estudio observamos que los internos VIH+ de uno de los reclusorios de la CM desarrollaron TB activa con características propias como una tasa de transmisión reciente con progresión rápida a enfermedad de $22 \%$, una prevalencia muy elevada $(16.3 \%)$, una incidencia alta (7.7 por 100 sujetos-año), y una proporción elevada de abandono del tratamiento $(18 \%)$. Varias características ayudan a entender esta situación: 1) se trata de individuos con infección avanzada por VIH; 2) la afección pulmonar fue la más frecuente $(75 \%)$, con retraso por más de tres meses en el diagnóstico y con mayor riesgo de transmisión, y 3) la mayoría de 


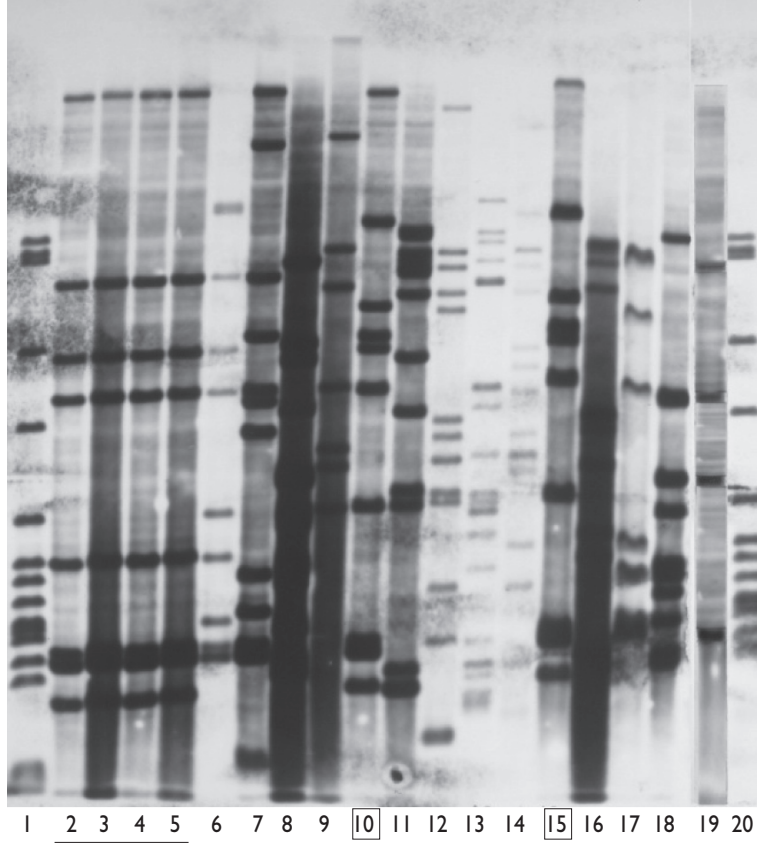

Figura 3. Tipificación por RFLP de los 18 aislados de MYCOBACTERIUM TUBERCULOSIS RECUPERADOS DE LOS INTERNOS CON TUBERCULOSIS ACTIVA EN UNA COHORTE DE RECLUSOS INFECtados por ViH en una cárcel de la Ciudad de México ATENDIDOS ENTRE NOVIEMBRE DE 2010 Y FEBRERO $201 \mathrm{I}$. EL CONGLOMERADO I CORRESPONDE A LOS CARRILES CON LOS NÚMEROS SUBRAYADOS Y EL CONGLOMERADO 2 A LOS ENCERRADOS EN UN CUADRO. LOS CARRILES I Y 20 CORRESPONDEN A LA CEPA de Referencia M. TUBerCulosis H37Rv los individuos refirieron también alcoholismo, uso de drogas y desnutrición.

Los reclusos suelen tener una prevalencia más elevada de TB en comparación con la comunidad. Recientemente, Fazel y Baillargeon ${ }^{16}$ reportaron prevalencias elevadas en países pobres. La mediana en 13 países de Europa Occidental fue de 90 casos por 100000 reclusos en 2002, mientras que en EUA (entre 1993 y 2003) se ha observado una prevalencia de 29.4 por cada 100000 reclusos en las cárceles federales y de 24.2 por 100000 en las estatales, en comparación con sólo 6.7 casos por 100000 en la población general. Estas tasas elevadas de TB son atribuibles a la concentración de factores de riesgo entre los reclusos (infección por $\mathrm{VIH}$, uso de drogas intravenosas, entre otras), aunado a las pobres condiciones de vida en las prisiones que facilitan la transmisión. Además los retrasos en el diagnóstico o en el tratamiento se han relacionado con diversos brotes. Por ello, los centros penitenciarios actúan como reservorios de la infección con riesgo de transmisión hacia las comunidades vecinas. ${ }^{16-18}$

Algunos otros ejemplos son: a) en cárceles españolas se encontró una tasa de incidencia de 2775 casos en 100000 reclusos, $77 \%$ con infección por $\mathrm{VIH}_{;}{ }^{17}$ b) en Bostwana fue de 3797 por 100 000, con $30 \%$ de coinfección, ${ }^{18}$ c) en Brasil de 2065 por 100 000, con $48.3 \%$ de coinfección, ${ }^{19}$ y d) en Nueva York (1990-1991), una tasa de incidencia de 156.2 casos por 100000 y coinfección en $95 \% .{ }^{20}$ En Tijuana, BC se observó una tasa de prevalencia de 2460 por 100000 y de coinfección en $7.6 \% .{ }^{21}$ Los resultados de la prisión de Tijuana y los

\begin{tabular}{|c|c|c|c|c|c|c|c|c|c|c|c|c|c|c|c|c|c|c|c|c|}
\hline & 2003 & 2004 & 2005 & 2006 & 2007 & & 08 & 2009 & ene-50 & feb- 10 & mar- 10 & $a b r-10$ & may-10 & jun-50 & jul-10 & ags- 10 & sep-10 & oct- 10 & nov- 10 & dic- 10 \\
\hline I & Sexoservidor & Cd Neza & & & & L 30 JII & i23-Sep & S Sep T Oct & & & & & & & 7 Fin $T x$ & & & & 212 & \\
\hline 2 & & & EUA & & Norte, 06 & & & & & & & $\mathrm{i} 30$ & & S4 & TI5 & L 17 & & & 161 & \\
\hline 3 & & & & & & Sexoser & & & & & & 16 Nte & $\mathrm{i} 29$ & S2I & $\mathrm{H} 28$ & T3 L 26 & & & 72 & \\
\hline 4 & & & Usuar & rio sex & o comercial & & & ur, $05-08$ & & & S20 & & 2 Ote & & il3 HI4 T2I & & & & 68 & \\
\hline
\end{tabular}

\begin{tabular}{|c|c|c|c|c|c|c|c|c|c|c|c|c|c|c|c|c|c|c|c|c|c|c|c|c|c|c|c|c|c|c|c|}
\hline & 1997 & 1998 & 1999 & 2000 & 2001 & 20022003 & 2004 & 2005 & 2006 & 2007 & 2008 & \multicolumn{10}{|c|}{2009} & \multicolumn{10}{|c|}{2010} \\
\hline & & & & & & & & & & & & ene & feb mar & $r$ abr may & y jun & jul & ago & $\operatorname{sep}$ & oct $n$ & $\operatorname{lov} d$ & dic & ene & feb ma & ar $a b_{1}$ & $\mathrm{r}$ may & y jun & jul & ag & sep & oct nov & $\mathrm{v}$ dic \\
\hline 1 & & & & & & il9/12 & & & & & & SI & DI/4 & & & & & & & & & & & & & & & & & 198 & \\
\hline 2 & Pto Vallarta & & Casa & Hogar & Pto $\mathrm{V}$ & allarta & Pto V & allarta & Sur, 02 & Lo8 & Sur, 03 il $8 / 04$ & & & & & & & & & & & & & & & & & & & 431 & \\
\hline
\end{tabular}

Estancia en SMA

Estancia en otro reclusorio

i Fecha de ingreso a SMA

$S$ Fecha de inicio de síntomas
$\mathrm{T}$ Fecha de inicio de tratamiento

$\mathrm{H}$ Fecha de hospitalización

L Libre

D Defunción

Figura 4. Se describen los grupos de aisLados idénticos en el patrón de RFLP. En el panel superior SE incluye las CARACTERÍSTICAS DEL CONGLOMERADO I. EN EL PANEL INFERIOR SE DESCRIBE EL SEgUNDO CONGLOMERADO. TODOS ELLOS INDIVIDUOS INFECTADOS POR VIH Y CON TUBERCULOSIS ACTIVA 
de este estudio muestran problemas de transmisión de $\mathrm{TB}$ en las prisiones mexicanas que ameritan un estudio profundo con el propósito de controlar la transmisión, particularmente hacia población de riesgo elevado $(\mathrm{VIH}+)^{22,23}$

De los reclusos con TB pulmonar, 78\% presentó alteraciones en la radiografía de tórax, lo que apoyaría su uso como método de escrutinio. A diferencia de otros reportes recientes, ${ }^{17,24}$ la baciloscopia y el cultivo fueron positivos en $90 \%$ de los casos con TB pulmonar. El beneficio del cultivo es grande porque permite realizar la susceptibilidad antimicrobiana y el análisis molecular. La transmisión reciente de TB se ha analizado en varios estudios en prisiones a través de genotipificación con IS6110. En este estudio encontramos dos conglomerados compuestos por seis aislados clínicos. Aun cuando no se pueda encontrar una liga epidemiológica de transmisión en todos los casos, al encontrar clonas idénticas con dos marcadores moleculares útiles, la única posibilidad plausible es que hayan sido resultado de un evento de transmisión. Así, cuatro casos fueron secundarios a transmisión reciente entre los 18 aislados tipificados, es decir, una tasa de transmisión reciente baja (22\%) en comparación con otras prisiones. En Nueva York reportaron como idénticos 31/39 aislados (79.5\%) de MDR-TB. ${ }^{20}$ En Barcelona 126/257 (51\%), con conglomerados integrados hasta por 25 sujetos y se identificaron 14 cadenas de transmisión que involucraron a 65 individuos (52\%). ${ }^{17}$ En Madrid 30/73 (41\%) formaron nueve conglomerados y se encontró parentesco epidemiológico en $37 \% .{ }^{25}$ En Brasil, 39 aislados se agruparon en seis conglomerados cuya información epidemiológica sugirió transmisión en la misma prisión. ${ }^{19}$

Al término de este estudio, solamente $2 / 7$ pacientes liberados acudieron a la clínica de referencia para la continuación del tratamiento, lo cual muestra falla en el seguimiento por parte de las autoridades de salud. En Costa de Marfil de 108 casos, 74.1\% curaron, 24\% fallecieron y $1.8 \%$ presentaron falla. ${ }^{26}$ En un estudio en Rusia sólo 26.3\%, de 80 reclusos liberados con TB activa, continuó su tratamiento. ${ }^{27}$ En Taiwán de 107 casos, $80.4 \%$ completó tratamiento, $15.9 \%$ perdió seguimiento al ser liberados y $3.7 \%$ murió. ${ }^{28}$ En España se ha demostrado que una buena coordinación entre los programas intra y extrapenitenciarios puede lograr una tasa elevada de adherencia al tratamiento $(97 \%$ en prisión y $79 \%$ en libertad). ${ }^{29}$ No encontramos resistencia antimicrobiana en este estudio en contraste con los reportes de otras series. ${ }^{8,30,31}$ Que la mayoría de los pacientes abandone el tratamiento al ser liberados es un factor de riesgo para el desarrollo de resistencia y de transmisión hacia la población general, por ello creemos que estos hallazgos deben alertar a las autoridades de salud y facilitar la coordinación en el manejo de los pacientes con TB o VIH una vez liberados.

Este estudio tiene varias limitaciones: 1) En diez casos contamos sólo con el expediente clínico, y no pudimos determinar los factores de riesgo; 2) En ocho casos no realizamos cultivo y no fue posible determinar transmisión reciente o resistencia; 3) Sólo se estudió a la población $\mathrm{VIH}+$ y no a todos los internos de esa prisión; la inclusión de toda la población de esta prisión ayudaría a un mejor entendimiento del fenómeno; 4) Los mecanismos de reinserción de estos individuos tienen debilidades, dado que se perdió $70 \%$; 5) Finalmente, el estudio de los pacientes no fue homogéneo por cuestiones logísticas, en algunos casos se realizó sólo biopsia de ganglio sin muestras respiratorias, por consiguiente no se pudo medir la carga de enfermedad en cada uno de ellos.

En conclusión, la frecuencia alarmante de TB en esta población de alto riesgo obliga a realizar un estudio más amplio para conocer la prevalencia general de TB en ésta y otras prisiones, con lo que se podrían mejorar los programas de control y seguimiento. El servicio médico penitenciario debe vincularse estrechamente con el programa nacional de control de TB para asegurar la adherencia al tratamiento de los pacientes liberados.

Declaración de conflicto de intereses. Los autores declararon no tener conflicto de intereses.

\section{Referencias}

I. Raviglione MC, Snider DE Jr, Kochi A. Global epidemiology of tuberculosis. Morbidity and mortality of a worldwide epidemic. JAMA 1995;273:220-226.

2. Horsburgh CR Jr. Priorities for the treatment of latent tuberculosis infection in the United States. N Engl J Med 2004;350:2060-2067.

3. Selwyn PA, Hartel D, Lewis VA, et al. A prospective study of the risk of tuberculosis among intravenous drug users with human immunodeficiency virus infection. N Engl J Med 1989;320:545-555.

4. Reyes $\mathrm{H}$. Pitfalls of TB management in prisons, revisited. Int J Prison Health 2007;3:43-67.

5. Tulsky JP, White MC, Dawson C, et al. Screening for tuberculosis in jail and clinic follow-up after release. Am J Public Health 1998;88:223-226.

6. Aerts A, Habouzit M, Mschiladze L, et al. Pulmonary tuberculosis in prisons of the ex-USSR state Georgia: results of a nation-wide prevalence survey among sentenced inmates. Int J Tuberc Lung Dis 2000;4: I I04- I I 10.

7. Slavuckij A, Sizaire V, Lobera L, et al. Decentralization of the DOTS programme within a Russian penitentiary system. How to ensure the continuity of tuberculosis treatment in pre-trial detention centres. Eur J Public Health 2002; 12:94-98.

8. Pleumpanupat W, jittimanee $S$, Akarasewi P, et al. Resistance to antituberculosis drugs among smear-positive cases in Thai prisons 2 years after the implementation of the DOTS strategy. Int J Tuberc Lung Dis 2003;7:472-477.

9. Jittimanee SX, Ngamtrairai N, White MC, Jittimanee S. A prevalence survey for smear-positive tuberculosis in Thai prisons. Int J Tuberc Lung Dis 2007; I 1:556-56I. 
10. Secretaría de Salud. Sistema Nacional de Vigilancia Epidemiológica 2008;25:I-3.

II. Modificación a la Norma Oficial Mexicana NOM-006-SSA2-1993, para la prevención y control de la tuberculosis en la atención primaria a la salud. Diario Oficial de la Federación; 27 de septiembre de 2005. Secretaría de Salud.

12. Nolte FS, Metchock B. Murray PR, Baron EJ, Pfaller MA, Tenover FC, et al. Manual of clinical microbiology. 6th. ed. Washington, DC: ASM Press, 1995:400-437.

13. Instituto Nacional de Diagnóstico y Referencia Epidemiológicas. Manual de Técnicas y Procedimientos de Laboratorio en Tuberculosis. México: Secretaría de Salud, 1992.

14. Van Embden JD, Cave MD, Crawford JT, et al. Strain identification of Mycobacterium tuberculosis by DNA fingerprinting: recommendations for a standardized methodology. J Clin Microbiol 1993;31:406-409.

15. Yang ZH, ljaz K, Bates JH, et al. Spoligotyping and polymorphic GCrich repetitive sequence fingerprinting of Mycobacterium tuberculosis strains having few copies of IS6I I O. J Clin Microbiol 2000;38:3572-3576. 16. Fazel S, Baillargeon J. The health of prisoners: Review. Lancet 20I I; 377: 956-965.

17. March F, Coll P, Guerrero RA, et al. Predictors of tuberculosis transmission in prisons: an analysis using conventional and molecular methods. AIDS 2000; 14:525-535.

18. Centers for Disease Control and Prevention (CDC). Rapid assessment of tuberculosis in a large prison system--Botswana, 2002. MMWR Morb Mortal Wkly Rep 2003;52:250-252.

19. Moreira-Oliveira MS, Oliveira HB, Pace F, et al. Molecular genotyping and epidemiology of Mycobacterium tuberculosis isolates obtained from inmates of correctional institutions of Campinas, Southeast Brazil. Braz J Infect Dis 2008; I 2:487-493.

20. Valway SE, Greifinger RB, Papania M, et al. Multidrug-resistant tuberculosis in the New York State prison system, 1990-1991. J Infect Dis 1994; 170:15I-156.

2I. Cerecer-Callú P, Aranda-Lozano JL, Márquez-Fiol AR, et al. Tuberculosis en un centro de readaptación social del noreste de México: estudio retrospectivo periodo 1999-2000, Tijuana, Baja California. Enf Inf Microbiol 2006;26:94-100

22. García-García ML, Sifuentes-Osornio J, Jiménez-Corona ME, et al. Drug resistance of Mycobacterium tuberculosis in Orizaba, Veracruz. Implications for the tuberculosis prevention and control program. Rev Invest Clin 2001;53:315-323.

23. Mohammad Z, Naing NN. Characteristics of HIV-infected tuberculosis patients in Kota Bharu Hospital, Kelantan from 1998 to $200 \mathrm{I}$. Southeast Asian J Trop Med Public Health 2004;35: I 40- 143.

24. Rutta E, Mutasingwa D, Ngallaba S, Mwansasu A. Tuberculosis in a prison population in Mwanza, Tanzania (1994-1997). Int J Tuberc Lung Dis $2001 ; 5: 703-706$

25. Fernández Jl, Fernández $\mathrm{K}$, Catalán S, et al. Transmisión de la tuberculosis en las prisiones de Madrid. Med Clin 2000; I 15:246-250.

26. Koffi N, Ngom AK, Aka-Danguy E, et al. Smear positive pulmonary tuberculosis in a prison setting: experience in the penal camp of Bouake, Ivory Coast. Int J Tuberc Lung Dis 1997; I:250-253.

27. Fry RS, Khoshnood K, Vdovichenko E, et al. Barriers to completion of tuberculosis treatment among prisoners and former prisoners in $\mathrm{St}$. Petersburg, Russia. Int J Tuberc Lung Dis 2005;9: 1027-1033.

28. Chiang CY, Hsu CJ, Hsu PK, et al. Pulmonary tuberculosis in the Taiwanese prison population. J Formos Med Assoc 2002;101:537-541. 29. Marco A, Caylà JA, Serra M, et al. Predictors of adherence to tuberculosis treatment in a supervised therapy programme for prisoners before and after release. Study Group of Adherence to Tuberculosis Treatment of Prisoners. Eur Respir J 1998; |2:967-97|.

30. Ruddy M, Balabanova Y, Graham C, et al. Rates of drug resistance and risk factor analysis in civilian and prison patients with tuberculosis in Samara Region, Russia. Thorax 2005;60:130-135.

31. Spradling P, Nemtsova E, Aptekar T, et al. Anti-tuberculosis drug resistance in community and prison patients, Orel Oblast, Russian Federation. Int J Tuberc Lung Dis 2002;6:757-762. 